\title{
PROPERTIES OF STRONGLY INTERACTING MATTER AND SEARCH FOR A MIXED PHASE AT THE JINR NUCLOTRON
}

\author{
A. N. Sissakian ${ }^{a}$, A. S. Sorin ${ }^{a, 1}$, M. K. Suleymanov ${ }^{a}$, \\ V.D. Toneev ${ }^{a}$, G. M. Zinovjev ${ }^{b}$ \\ ${ }^{a}$ Joint Institute for Nuclear Research, Dubna \\ ${ }^{b}$ BITP NAS, Kiev, Ukraine
}

A physical program is formulated for new facilities opening in Dubna for acceleration of heavy ions with an energy up to $5 A \mathrm{GeV}$.

Сформулирована программа физических исследований, которые могут быть проведены в Дубне благодаря открывающимся новым возможностям по ускорению тяжелых ионов с энергиями до $5 A$ Гэ $\mathrm{B}$.

PACS: $12.38 .-\mathrm{t}$

\section{INTRODUCTION}

High-multiplicity physics serves as a bridge which joins the study of high-energy mechanisms of particle production and that of new phases of matter. The latter is of great interest for relativistic nuclear collisions. Over the last 25 years a lot of efforts have been made to search for new states of strongly interacting matter under extreme conditions of high temperature and/or baryon density, as predicted by QCD. These states are relevant to understanding of the evolution of the early Universe after Big Bang, the formation of neutron stars and the physics of heavy-ion collisions. Heavy-ion collisions are of great importance since they open a way to reproduce these extreme conditions in the Earth laboratory. This explains a permanent trend of the leading world research centers to construct new heavy-ion accelerators for even higher colliding energy.

Looking at the list of heavy-ion accelerators, one can see that after the first experiments at the Dubna Synchrophasotron, heavy-ion physics successfully developed at Bevalac (Berkeley) with the bombarding energy to $E_{\mathrm{lab}} \sim 2 A \mathrm{GeV}$, AGS (Brookhaven) $-E_{\mathrm{lab}} \sim 11 A \mathrm{GeV}$, and SPS $(\mathrm{CERN})-E_{\text {lab }} \sim 160 A \mathrm{GeV}$. The first two machines are closed now. The nuclear physical programs at SPS as well as at SIS (GSI, Darmstadt, $E_{\text {lab }} \sim 1 A \mathrm{GeV}$ ) are practically completed. The new relativistic heavy-ion collider (RHIC, Brookhaven) is intensively working

\footnotetext{
${ }^{1}$ E-mail: sorin@theor.jinr.ru
} 
in the ultrarelativistic energy range $\sqrt{s_{N N}} \sim 200 \mathrm{GeV}$ for searching signals of the quarkgluon plasma formation. In this respect, many hopes are related to the Large Hadron Collider (LHC, CERN) which will start to work in the TeV region in two-three years. The lowenergy scanning program at SPS (NA49 Collaboration) revealed an interesting structure in the energy dependence of some observables at $E_{\text {lab }} \sim 20-30 A \mathrm{GeV}$, which can be associated with the exit of an excited system into a decofinement state. This fact essentially stimulated a new large project FAIR GSI (Darmstadt) for studying compressed baryonic matter in a large energy range of $E_{\mathrm{lab}}=10-30 \mathrm{~A} \mathrm{GeV}$ which should come into operation in 2013.

On the other hand, at JINR there is a modern superconducting accelerator, Nuclotron, which has not realized its planned parameters yet. In this paper we would like to present arguments that acceleration of heavy ions like $\mathrm{Au}$ at the Nuclotron up to the maximal planned energy $E_{\text {lab }}=5 \mathrm{~A} \mathrm{GeV}$ allows one to study properties of hot and dense nuclear matter to be competitive at the world level.

\section{POSSIBLE STATES OF STRONGLY INTERACTING MATTER}

A convenient way to present a variety of possible states of strongly interacting matter is a phase diagram in terms of temperature $T$ and baryon chemical potential $\mu_{B}$ (or baryon density $n_{B}$ ), as shown in Fig. 1. From this schematic picture it is seen in which region of the diagram the given phase is realized and which colliding energies are needed to populate
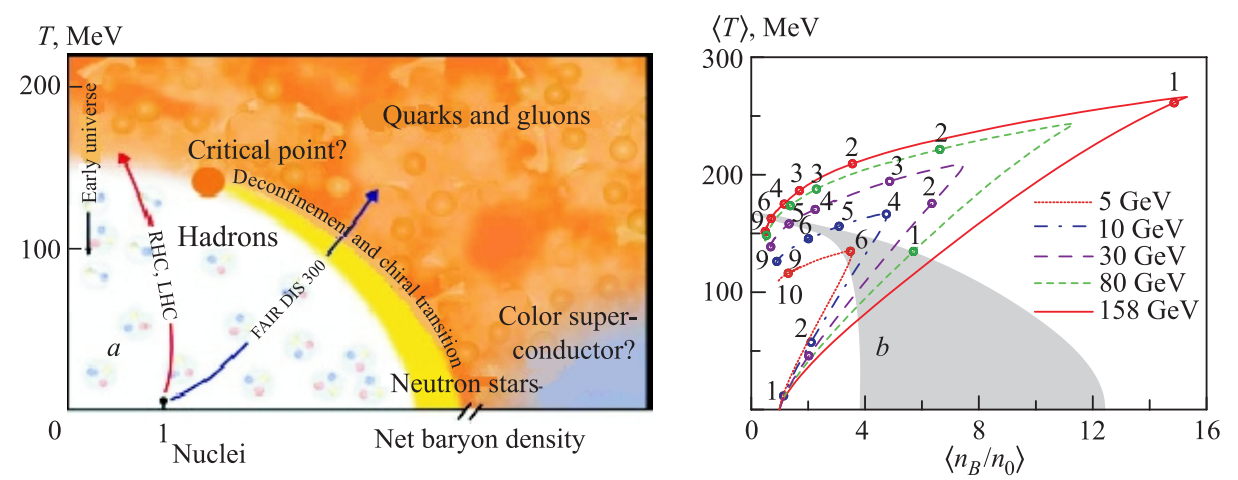

Fig. 1. Artist's view of the phase diagram [1] $(a)$ and dynamical trajectories for central $\mathrm{Au}+\mathrm{Au}$ collisions in $T-n_{B}$ plane (b) for various bombarding energies calculated within the relativistic 3-fluid hydrodynamics [2]. Numbers near the trajectories are the evolution time moments

this region. In particular, the boundary of the deconfinement and chiral symmetry restoration transition may be reached even below the energies planned in the FAIR GSI project but the nuclear matter compression should be high enough.

This point is illustrated in more detail in Fig. 1, $b$.

As is seen, a system, formed in a high-energy collision, is fast heated and compressed and then starts to expand slowly reaching the freeze-out point which defines observable hadron quantities. Indeed, at the maximal achievable Nuclotron energy $E_{\text {lab }}=5 \mathrm{~A} \mathrm{GeV}$ the system «looks» into the mixed phase for a short time. However, uncertainties of these calculations are still large. If heavy masses for quarks are assumed, the phase boundary is shifted towards 
higher $n_{B}$ as compared to those shown in Fig. 1. On the other hand, dynamical trajectories are calculated for a pure hadronic gas equation of state, and the presence of a phase transition may noticeably change them. In addition, near the phase transition the strongly interacting system behaves like a liquid rather than a gas, as has been recently clarified at small $\mu_{B}$ [3]. As to high $n_{B}$ values, it is a completely open question. It is hard to believe that some irregular structure like that at $E_{\text {lab }} \sim 30 \mathrm{~A} \mathrm{GeV} \mathrm{[4]} \mathrm{can} \mathrm{manifest} \mathrm{itself} \mathrm{at} \mathrm{the} \mathrm{Nuclotron} \mathrm{energy.} \mathrm{So,}$ the global observables (average multiplicities, rapidity spectra, transverse distributions and so on) are expected to be quite smooth with energy. However, it might not be the case for more delicate characteristics. In any case, due to the proximity of the phase diagram region under discussion to the confinement transition and chiral symmetry restoration, some precursory phenomena cannot be excluded at a bombarding energy about $5 \mathrm{~A} \mathrm{GeV}$, which opens a new perspective for physical investigations at the Dubna Nuclotron.

\section{ARGUMENTS}

Below, some arguments in favor of this statement are given:

- Properties of hadrons are expected to change in hot and/or dense baryon matter $[5,6]$. This change concerns hadronic masses and widths, first of all for the $\sigma$ meson as the chiral partner of pions, which characterizes a degree of chiral symmetry violation and can serve as a «signal» of its restoration as well as the mixed phase formation. Lepton decays in matter of vector mesons (particularly $\rho$ and $\omega$ ) are also very attractive.

The presence of in-medium modification of $\rho$ mesons has been proved in the CERES experiments (see Fig. 2). The observed essential enhancement of low mass $(0.2 \lesssim M \lesssim 0.7)$ lepton pairs, as compared to free hadron decays, is due to the influence of hot and dense nuclear matter on properties of the $\rho$-meson spectral function. Unfortunately, poor resolution in the di-electron mass does not allow discrimination of different physical scenarios of this effect in the CERES experiments.

As to in-medium $\sigma$-meson decay, some indications were obtained in reactions induced by pions and $\gamma$ 's [9-11]. In Fig. 3, the relative pion pair abundance $C_{\pi \pi}^{A}$, defined as

$$
C_{\pi \pi}^{A}=\frac{\sigma^{A}\left(M_{\pi \pi}\right)}{\sigma_{T}^{A}} / \frac{\sigma^{N}\left(M_{\pi \pi}\right)}{\sigma_{T}^{N}},
$$

is presented for different isotopic states of observed pions vs the invariant pion pair mass $M_{\pi \pi}$.

A sizable enhancement is observed at low $M_{\pi \pi}$ in the case of $\pi^{+} \rightarrow \pi^{+} \pi^{-}$reaction, but not for the $\pi^{+} \rightarrow \pi^{+} \pi^{+}$case [9]. This enhancement is related to a possible $\pi \pi$ scattering in a nucleus via formation of a virtual scalar $\sigma$ meson, which is forbidden for the $\pi^{+} \pi^{+}$ state. The effect is getting stronger for heavier nuclei. Recently, using ELSA tagged facilities in Bonn, the in-medium modification of the $\omega$ meson has first been observed in reaction $\gamma+A \rightarrow \omega+X \rightarrow \pi^{0}+\gamma+X$ [11]. A small shift in the $\omega$ mass was detected. Note, however, that in $\pi$ - and $\gamma$-induced reactions we deal with comparatively low baryon density states, $n_{B} \sim(1-2) n_{0}$. There are no similar experiments with heavy ions.

Nevertheless, there are theoretical proposals to probe chiral symmetry restoration in the vicinity of the phase transition boundary. In particular, it was shown $[12,13]$ that a twophoton decay of the $\sigma$ meson formed as an intermediate state in $\pi \pi$ scattering may be a very 


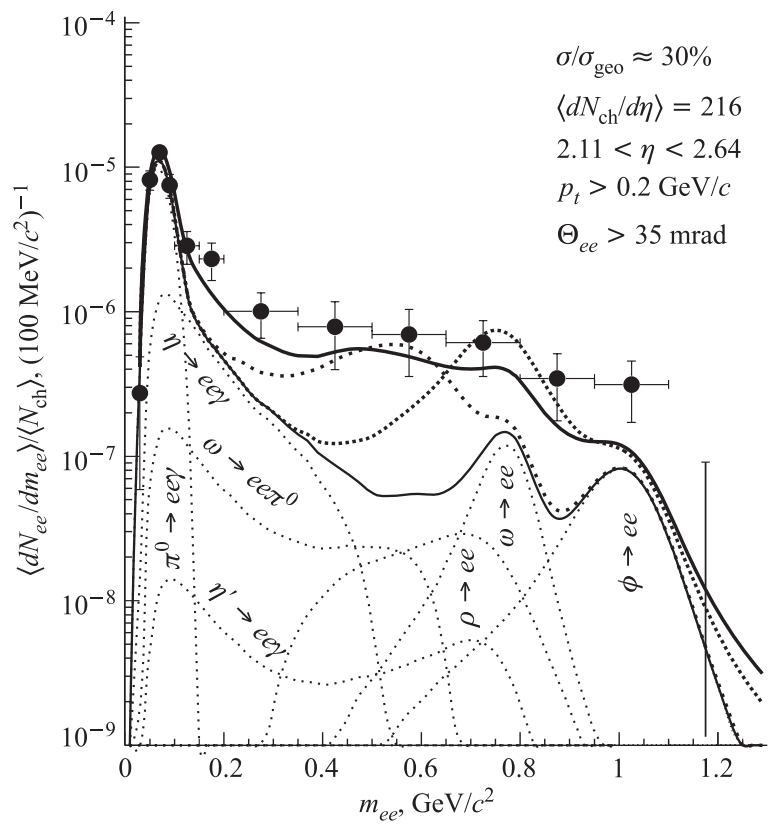

Fig. 2. The $e^{+} e^{-}$invariant spectra from central $\mathrm{Pb}+\mathrm{Au}(40 A \mathrm{GeV})$ collisions [7]. Thin solid and dotted lines are hadronic cocktail and calculation results for free $\rho$ mesons, respectively. Appropriate thick solid and dash-dotted lines are calculated in the Rapp-Wambach [8] and Brown-Rho [6] scenarios. Contributions of different channels are shown as well

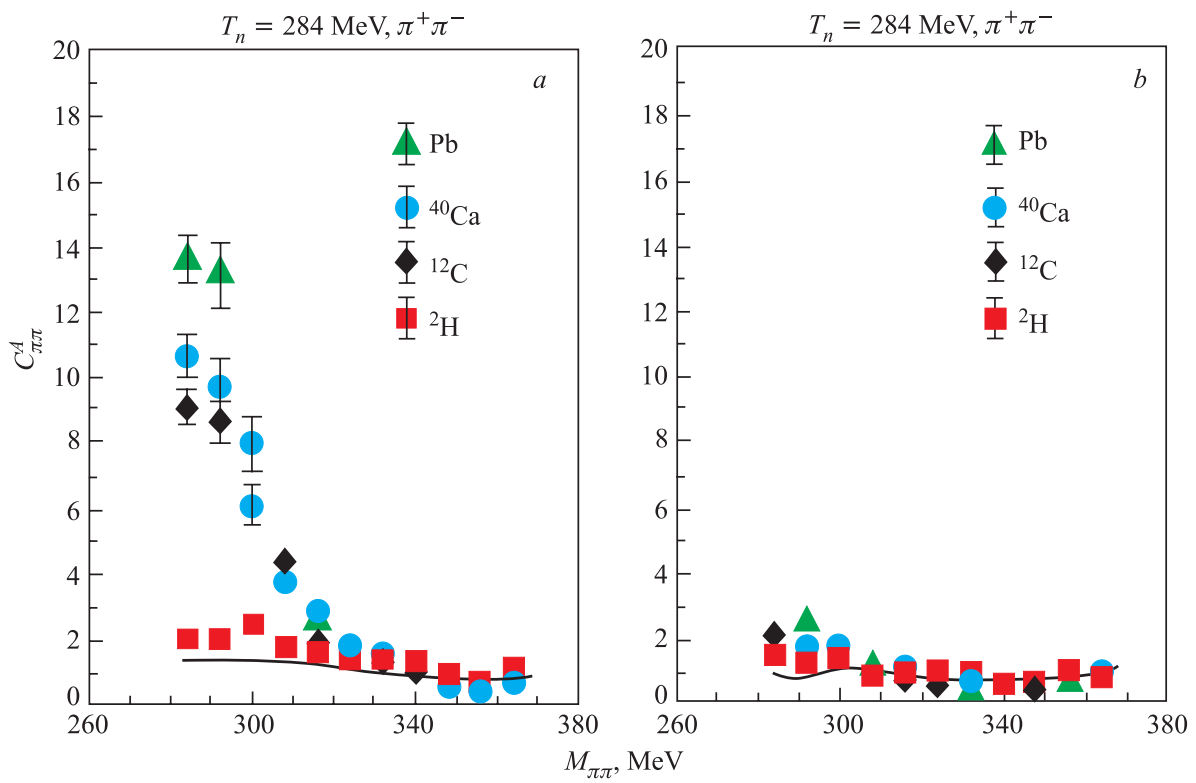

Fig. 3. Invariant mass distribution of pion pairs from $\pi$-nucleus interactions [9] 


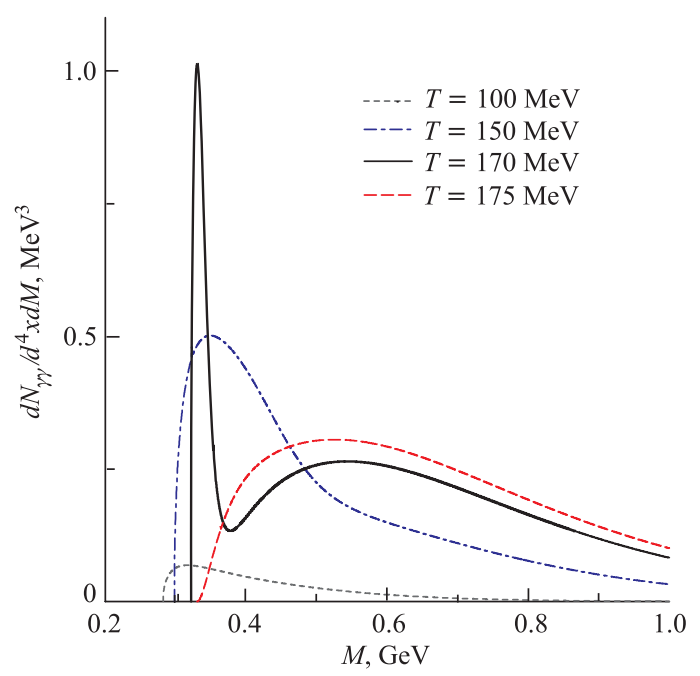

Fig. 4. Invariant mass spectra of $2 \gamma$ at $\mu_{B}=80 \mathrm{MeV}$ and different temperatures [13]

attractive signal. As pictured in Fig. 4, at temperature in the vicinity of the phase transition, when $m_{\sigma} \sim 2 m_{\pi}$, there is an anomalous peak in invariant mass spectra of $\gamma$ pairs which may serve as a signal of the phase transition and formation of a mixed phase. Certainly, there is a huge combinatorial background due to $\pi^{0} \rightarrow \gamma \gamma$ decays, but the Nuclotron energy is expected to have some advantage against higher energy accelerators because the contribution of deconfined quarks-gluons will be negligible.

This effect may be observed in the $e^{+} e^{-}$decay channel as well [14].

- Electromagnetic probes discussed above carry out information concerning the whole evolution of colliding nuclei and states which are realized at that. The bulk of produced hadrons is related to the freeze-out point where information on the interaction dynamics has been essentially erased. So, the expected behavior of global hadronic observables is smooth. However, some peculiarities of delicate hadron characteristics may be found and their hints are available even now.

In Fig. 5, the beam energy dependence of the elliptic flow coefficient $v_{2}$ is presented for protons in the midrapidity range from noncentral heavy-ion collisions [15]. The elliptic flow characterizes the angular anisotropy in the transverse momentum plane as

$$
\frac{d N}{d \phi} \sim\left[1+2 v_{1} \cos (\phi)+2 v_{2} \cos (2 \phi)\right]
$$

where the $\phi$ angle is measured from the reaction plane. As is seen, just near the Nuclotron energy $E_{\text {lab }} \sim 5 A \mathrm{GeV}$ the coefficient $v_{2}$ changes its sign and the transverse flow evolves from the out-of-plane towards in-plane emission. This fact may be treated as softening of the equation of state to be a precursor of the phase transition. The elliptic flow shows an essentially linear dependence on the impact parameter with a negative slope at $E_{\text {lab }}=2 A \mathrm{GeV}$, with a positive slope at $6 A \mathrm{GeV}$ and with a near-zero slope at $4 A \mathrm{GeV}$ [16]. This dependence serves as an important constraint to high-density behavior of nuclear matter for discrimination of various equations of state. 


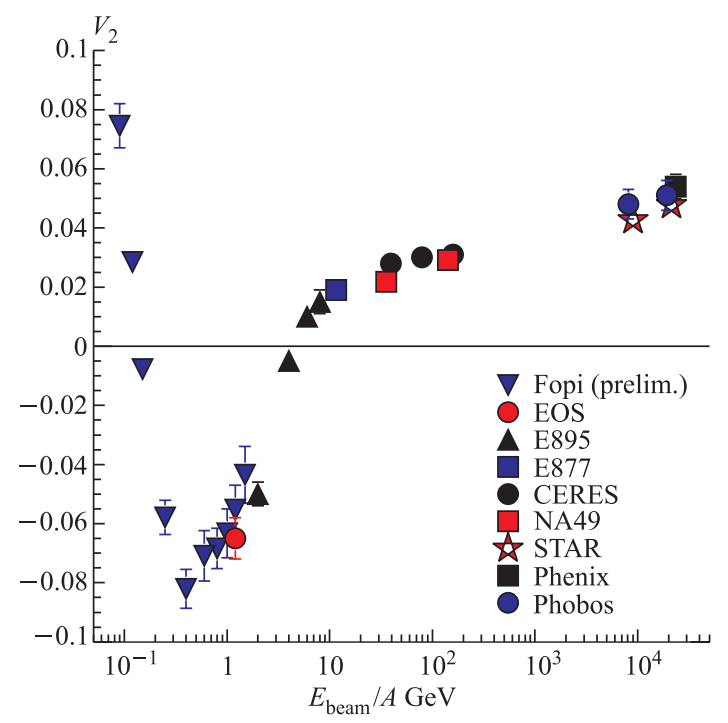

Fig. 5. Excitation function of the proton elliptic flow [15]

- Strangeness enhancement is an intriguing point of physics of heavy-ion collisions, being one of the first proposed signals of quark-gluon plasma formation. An important experimental finding is the observation of some structure («horn») in the energy dependence of reduced strangeness multiplicity at $E_{\text {lab }} \sim 30 \mathrm{~A} \mathrm{GeV}$, predicted in [17] as a signal that the formed excited system came into a deconfinement phase. As an example, in Fig. 6 the $K^{+} / \pi^{+}$ average multiplicity ratio is displayed as a function of the bombarding energy. The «horn»

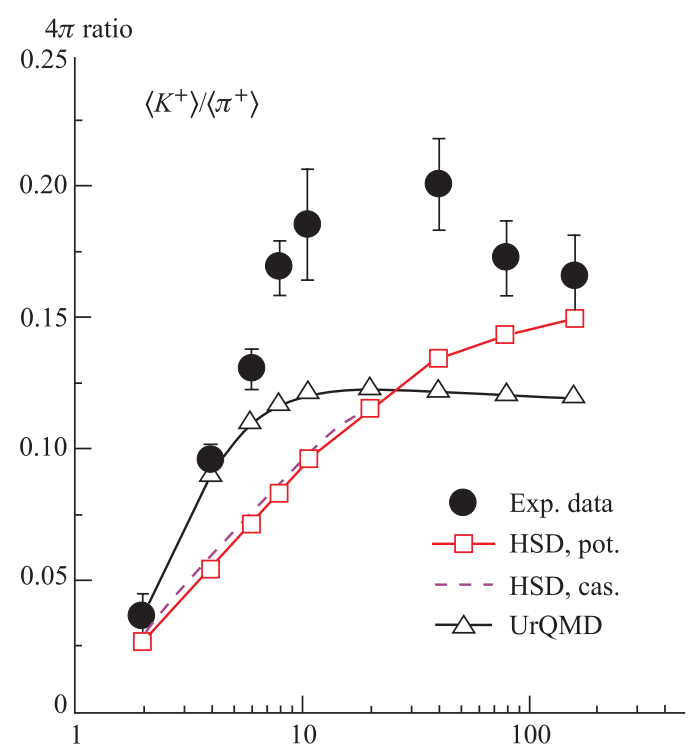

Fig. 6. Energy dependence of the relative strangeness abundance of $K^{+}$mesons [18]. Curves are results of different transport calculations 
structure is well visible and is getting even more prominent if the recent measurement of $\left\langle K^{+}\right\rangle /\left\langle\pi^{+}\right\rangle \simeq 0.22$ at $E_{\text {lab }}=20 A \mathrm{GeV}$ [4] is additionally plotted and the bombarding energy is presented in the logarithmic scale (which is quite natural when RHIC data are supplemented). It is of great interest that these global characteristics are not explained by modern transport theory (UrQMD, HSD models). While the average pion and kaon multiplicities are well reproduced at the SIS and SPS energies, the above-mentioned models essentially underestimate the $K / \pi$ ratio in the AGS energy domain. It is remarkable that the divergence between the transport theory and experiment starts just at the Nuclotron energy. This increases interest in future experiments at the Nuclotron.

- One should note that peculiarities at the same «horn» energy are observed also in other measured quantities [4]. In particular, the reduced pion multiplicity $\left\langle n_{\pi}\right\rangle / A_{\text {part }}$ for nucleus-nucleus collisions is below that for elementary $N N$ interactions at moderate energies and exceeds appropriate $N N$ values in a relativistic domain. According to $[4,17,19]$, the equality of the nuclear and elementary $\left\langle n_{\pi}\right\rangle / A_{\text {part }}$ ratios at the «horn» energy is treated as an argument in favor of the onset of a deconfinement. However, this proximity of nuclear and elementary reduced pion multiplicities starts at the Dubna Nuclotron energy and could naturally be explained by the role of the $\Delta$ isobar in the pion absorption. To clarify this or alternative interaction mechanisms, new experimental data for heavy-ion collisions with scanning over the Nuclotron energy range are needed.

- Existing experimental data indicate a large degree of equilibration of nuclear matter in high-energy heavy-ion collisions (see review article [20]). The mechanisms of equilibration are still unclear. In particular, a large difference between dynamical and statistical models is observed for heavy baryons (e.g., for multi-strange hyperons, $\Xi$ and $\Omega^{-}$and resonances) measured at high energies. The role of multiparticle interactions is also obscure. The corresponding data for the Nuclotron energies are extremely poor. Future Nuclotron measurements could give a strong input for a further development of dynamical and statistical approaches and allow one to disentangle these two types of models.

To a certain extent, the study of fluctuations in relativistic strongly interacting matter may help in solving the equilibration problem. Experimental data on event-by-event fluctuations (e.g., fluctuations of particle multiplicity, electric, baryon and strangeness charges) in nuclear collisions give a unique possibility to test recent ideas on the origin of fluctuations in relativistic interacting systems [21]. Measurements require tracking detectors of large acceptance and precise control of collision centrality on event-by-event basis. Up to now only results on very limited acceptance at high energies are available, thus new measurements at the Nuclotron energy are of particular importance. From the experimental point of view the Nuclotron energy range seems to be ideal for these measurements. This is because moderate particle multiplicity and relatively broad angular distribution simplify an efficient detection of all produced charged particles.

An investigation of the narrow femtoscopic correlations [22] joins this problem. The femtoscopic analysis exploits particle correlations at small relative velocities and is widely used to study space-time characteristics (the system size, evolution time, freeze-out duration) of the production processes in relativistic heavy-ion collisions. There is substantial experience accumulated at JINR in the field of particle correlations. Such femtoscopic data are missing at the Nuclotron energies.

One should emphasize that all these investigations suppose that centrality of heavy-ion 
collisions is under control and centrality scanning of the characteristics under discussion is an indispensable condition.

Measurements of these quantities at the Nuclotron energies should be considered as a necessary continuation of global efforts to establish the energy dependence of properties of hadron production and search for signals of a phase change in nuclear collisions.

\section{THEORETICAL AND EXPERIMENTAL STUDIES AT JINR}

In this regard the following theoretical and experimental studies at JINR are considered as perspective:

1) investigation of the hadron properties in hot and/or dense baryonic matter. A spectral function change is expected, first of all for the $\sigma$ meson as a chiral partner of pions, which characterizes a degree of chiral symmetry violation. The rare specific channels of $\rho$-meson decays are also quite attractive.

Solving these issues assumes a proper understanding of reaction mechanisms of highenergy colliding ions, knowledge of properties of interacting matter and its equation of state. In this respect more general research is in order;

2) analysis of multiparticle hadron interactions, targeted at the development of new statistical treatment as well as codes for space-time evolution of heavy nuclei collisions at high energies. Particular attention should be paid to signals of new phase formation during this evolution;

3) study of the system size, evolution time, freeze-out duration in the femtoscopic analysis (noticeable volume expansion is expected if the mixed phase is formed), scanning in atomic number and energy;

4) study of the energy and centrality dependences of the pion, hadron resonance and strange particle multiplicities, and the ratio of their yields, together with the transverse momentum, including $K^{-}-, K^{*}$ - and $\phi$-meson spectra as well as manifestation of baryon repulsion effects on hadron abundances;

5) study of dilepton (electron and muon pairs) production to see in-medium modification of hadron properties at high baryon densities;

6) study of angular correlations in the transverse plane as well as radial, directed and elliptic flows;

7) fluctuation study of particle multiplicities, electric charges and transverse momenta (their energy dependences could give information on the phase transition range);

8) study of nuclear fragments characteristics vs centrality (change of behavior compared to the peripheral collisions is expected), universality of nuclear fragmentation;

9) energy and atomic number scan all characteristics of central collisions of heavy nuclei (this might allow one to obtain information on the equation of state of strongly interacting matter in the transition region), difference between central collisions of light nuclei and peripheral collisions of heavy ions.

\section{CONCLUSION}

The JINR Nuclotron has a possibility to accelerate heavy ions (up to $A>200$ ) to the maximal energy of $5 \mathrm{~A} \mathrm{GeV}$ in about two years. This gives a chance to address experimentally 
many recent problems within the next several years before the FAIR GSI accelerator come into operation. The proposed research program at the Nuclotron may be also considered as a pilot study preparing for subsequent detailed investigations at SIS-100/300 [1] and as an integral part of the world scientific cooperation to study the energy dependence of hadron production properties in nuclear collisions.

Acknowledgements. We would like to thank members of the working group N. Amelin, K. Abramyan, D. Blaschke, Yu. Bystritsky, Yu. Kalinovsky, V. Karnaukhov, A. Kovalenko, V. Krasnov, E. Kuraev, A. Kurepin, R. Lednicky, J. Lukstins, A. Malakhov, I. Molodtsova, S. Molodtsov, A. Radzhabov, V. Skokov, O. Teryaev, A. Vodopianov, M. Volkov and V. Yudichev for a fruitful collaboration. We greatly appreciate many useful and valuable discussions with M. Gazdzicki, M. Gorenstein, H. Gutbrod, T. Hatsuda, T. Kunihiro, H. Satz and H. Ströbele. We also would like to express special thanks to A. Filippov, V. Voronov and V.Zhuravlev for support during these investigations. This work was supported in part by RFBR Grant No. 05-02-17695 and by a special program of the Ministry of Education and Science of the Russian Federation (grant RNP.2.1.1.5409).

\section{REFERENCES}

1. Proposal for an International Accelerator Facility for Research with Heavy Ions and Antiprotons. http://www.gsi.de/documents/DOC-2004-Mar-196-2.pdf

2. Ivanov Y. B., Russkikh V. N., Toneev V. D. // Phys. Rev. C. 2006. V.73. P. 044904; nucl-th/0503088.

3. Shuryak E. V., Zahed I. hep-ph/0307267;

Brown G. E., Lee Ch.-H., Rho M. hep-ph/0402207;

Voskresensky D. N. // Nucl. Phys. A. 2004. V.744. P. 378; hep-ph/0402020.

4. Gazdzicki M. nucl-ex/0507017;

Blume C. (NA49 Collab.). hep-ph/0505137.

5. Hatsuda T., Kunihiro T. // Phys. Rep. 1994. V.247. P. 221.

6. Brown G. E., Rho M. // Phys. Rep. 1996. V. 269. P. 333.

7. Adamova D. et al. (CERES/NA45 Collab.). nucl-ex/0209024.

8. Rapp R., Wambach J. // Adv. Nucl. Phys. 2000. V.25. P.1.

9. Bonutti F. et al. (CHAOS Collab.) // Phys. Rev. Lett. 1996. V.77. P.603; Nucl. Phys. A. 2000. V. 677. P. 213.

10. Starostin A. et al. (Crystal Ball Collab.) // Phys. Rev. Lett. 2000. V.85. P. 5539.

11. CBELSA/TAPS Collab. nucl-ex/0504010.

12. Chiku S., Hatsuda T. // Phys. Rev. D. 1998. V. 58. P. 076001; hep-ph/9809215; Hatsuda T., Kunihiro T., Shimizu H. // Phys. Rev. Lett. 1999. V. 82. P. 2840.

13. Volkov M. K. et al. // Phys. Lett. B. 1998. V.424. P. 235; hep-ph/9706350.

14. Weldon H. A. // Phys. Lett. B. 1992. V.274. P. 133. 
15. Stock $R$. nucl-ex/0405007.

16. Chung P. et al. nucl-ex/ 0112002.

17. Gazdzicki V., Gorenstein M. I. // Acta Phys. Polon. B. 1999. V. 30. P. 2705; hep-ph/9803462.

18. Weber H. et al. nucl-th/0209079.

19. Senger P., Ströbele H. // J. Phys. G: Nucl. Part. Phys. 1999. V.25. P. R59.

20. Braun-Munzinger P., Redlich K., Stachel J. Quark Gluon Plasma 3 / Eds. Hwa R.C., Wang X. N. Singapore, 2004. P. 491; nucl-th/0304013.

21. Begun V. V. et al. // Phys. Rev. C. 2005. V.71. P. 054904;

Keranen A. et al. // J. Phys. J. 2005. V.31. P. S1095;

Begun V. V. et al. // Phys. Rev. C. 2005. V.71. P. 054904.

22. Wiedemann U.A., Heinz U. // Phys. Rep. 1999. V.319. P. 145;

Csorgo T. // Heavy Ion Phys. 2002. V. 15. P. 1;

Lednicky R. // Phys. At. Nucl. 2004. V. 67. P.72; Nucl. Phys. A. 2006. V.774. P. 189;

Lisa M. et al. // Ann. Rev. Nucl. Part. Sci. 2005. V.55. P.37.

Received on September 21, 2006. 